\title{
PROJETO DE ILUMINAÇÃO NATURAL: FERRAMENTAS PARA CÁLCULO E AVALIAÇÃO
}

\author{
LIMA, Thais Borges Sanches; CHRISTAKOU, Evangelos Dimitrios.
}

\section{RESUMO}

As decisões tomadas pelo arquiteto nos estágios iniciais do projeto têm impacto decisivo no desempenho luminoso do edifício. Diversas são as ferramentas de apoio à concepção do projeto que podem ser aplicadas para o cálculo e avaliação da iluminação natural. Este artigo visa descrever a importância do uso dessas ferramentas para o cálculo e avaliação de projetos de iluminação natural. Para isso, foram descritas as vantagens e limitações encontradas na aplicação das ferramentas, dandoumenfoquemaioraousodasimulação computacional. Assim, para o estudo dos aspectos quantitativos, as ferramentas convencionais (métodos matemáticos e gráficos) são as mais aplicadas. Os modelos em escala, utilizados por projetistas para a avaliação dos projetos, nem sempre são aproveitados para a análise da iluminação, apesar de permitir também a visualização do ambiente iluminado. As ferramentas computacionais permitem estudos tanto quantitativos como qualitativos e têm sido aplicadas para análise da qualidade ambiental de edificações, porém ainda são pouco utilizadas na prática profissional.

Palavras-chave: Projetação arquitetônica, Projeto de iluminação, Simulação computacional da iluminação.

\section{ABSTRACT}

Decisions made by the architect during the initial stages of design have a huge impact upon the building's luminous performance. There are several existing tools to aid the architect during design, which are capable of performing calculations and evaluating natural illumination. This paper illustrates the importance of applying such design tools for calculatingandevaluating natural illumination design. The advantages and limitations encountered during the application of such tools have been described in this article, with a greater emphasis upon computer simulation tools. As a result, in order to obtain quantitative results, conventional tools such as mathematical models and graphs, are the most used. Physical models, used by architects to visualize their design, are not commonly used as a tool for the evaluation of the internal and external daylight incidence upon the scaled model. Computer simulations allow both qualitative and quantitative studies and have been applied towards the analysis of the environmental performance of buildings, although little is used in the architectural practice.

Key words: Architectural Design, Lighting Design, Computer Simulation of Lighting

\section{INTRODUÇÃO}

O estudo da iluminação nos projetos de arquitetura deve ser desenvolvido ainda na fase de concepção da proposta projetual, visto que, grande parte das variáveis relacionadas ao aproveitamento da luz natural nos ambientes, tais como, orientação do edifício, posicionamento, tamanho e orientação das aberturas, é definida ainda nessa fase.

O projeto de iluminação é composto de três etapas principais, denominadas definição, cálculo e avaliação, desenvolvidas a partir de uma alternativa de solução, para atender às questões quantitativas, relacionadas ao nível de iluminação necessário para a execução das atividades a que o espaço se propõe; e às questões qualitativas para uma melhor apreensão do espaço do ponto de vista do conforto visual, devendo ser entendido como a integração da iluminação natural e artificial (LIMA, 2003).

A etapa de definição da proposta consiste na determinação das características do problema e as possíveis soluções. Na etapa de cálculo são definidos os parâmetros quantitativos através de um modelo matemático da iluminação dos ambientes. 
A avaliação é a etapa onde se verifica se a solução proposta atende aos requisitos estabelecidos para o problema.

Os instrumentos para projeto de iluminação são utilizados nas etapas de cálculo e avaliação e são divididos, segundo Baker e Steemers (1998), em 3 categorias: modelos em escala, ferramentas de projeto simplificadas e ferramentas computacionais.

As duas primeiras categorias podem ser definidas como ferramentas convencionais, embora os modelos em escala, muito usados para apresentar as propostas projetuais, sem sempre são aplicados para o estudo e análise do projeto de iluminação natural (BRYAN et al, 1981, apud PEREIRA, 1995). As ferramentas computacionais têm ganhado espaço com o uso de programas de simulação, simples ou complexos, que visam analisar os aspectos quantitativos e qualitativos da utilização da luz natural.

Os procedimentos de cálculo convencionais podem ser encontrados em publicações específicas e na norma NBR 15215 (ABNT, 2005), que descreve os métodos para cálculo da iluminação natural e para verificação experimental das condições de iluminância e luminância em ambientes internos.

As técnicas de simulação computacional estão sendo utilizadas, principalmente nos ambientes acadêmicos, onde estudos têm avaliado sua aplicabilidade no desenvolvimento de projetos de iluminação.

Este artigo visa descrever as ferramentas de projeto de iluminação naturala, destacando suas potencialidades $\mathrm{e}$ limitações, dando ênfase às ferramentas de simulação computacional, visto que estas têm possibilitado análises mais complexas, principalmente em relação aos aspectos qualitativos do conforto visual.

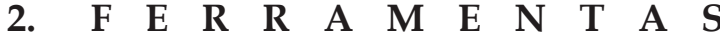 CONVENCIONAIS}

As chamadas ferramentas convencionais a muito tem sido utilizadas no desenvolvimento de projetos de iluminação. São baseadas em métodos analíticos de avaliação dos níveis de iluminação do ambiente, mas não possibilitam, principalmenteasferramentas simplificadas, a análise com relação aos aspectos qualitativos da iluminação.

\subsection{Modelos físicos em escala reduzida}

Os modelos físicos em escala reduzida têm uma série de vantagens com relação ao estudo da iluminação natural, mas também possuem algumas limitações, dentre as quais podemos destacar: a necessidade de precisão na geometria das obstruções e exposição ao céu e a interferência causada pelo observador ou câmera no nível luminoso resultante (MOORE, 1985).

Pereira (1995) destaca ainda a limitação com relação a estudos paramétricos, a necessidade de boa instrumentação fotométrica, além de demandar bastante tempo para a construção do modelo.

Existem duas formas de simular a iluminação natural nos modelos em escala: o uso do céu real, que é mais barato e fácil, porém implica em condições de estudo muito variáveis e de pouco controle; e o uso de céu artificial, onde as condições são estáveis, porém tendo a limitação de ser usado somente para estudos sob céu nublado e de tamanho finito (MOORE, 1985) (Figura 1).

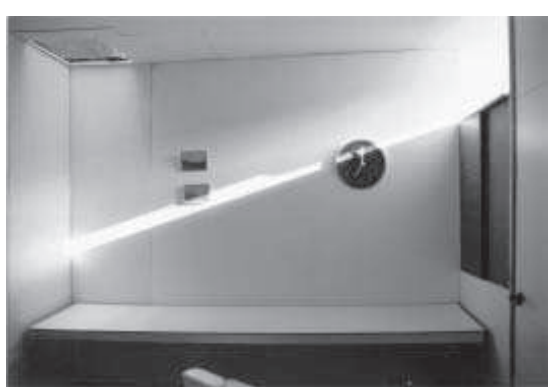

Figura 1 - Estudo de iluminação com modelo em escala reduzida ${ }^{\mathrm{b}}$

Com relação às vantagens de sua aplicação, a maior delas se relaciona à dimensão do modelo, que considerando a incidência da luz natural e sua análise, não vai influenciar nos resultados, visto que o 
comportamento é o mesmo, independente da escala utilizada. Esta só irá influenciar em análises com o uso de fotocélulas e câmeras fotográficas, que requerem dimensões apropriadas para sua aplicação no interior do modelo.

Apesar disso, os modelos físicos em escala oferecem precisão nos resultados quantitativos e facilidade de comparação para mudanças de umúnico componente de projeto; fornece uma percepção do espaço iluminado e, portanto a possibilidade de estudos qualitativos; permite simular formas mais complexas; e visualizar as mudanças ocorridas com a variabilidade da luz natural durante o dia; além de que, a maioria dos projetistas tem familiaridade no uso desses modelos (MOORE, 1985; BAKER e STEEMERS, 1998).

\subsection{Ferramentas de projeto simplificadas}

As ferramentas de projeto simplificadas ainda são as mais utilizadas por projetistas que desenvolvem projetos de iluminação, tanto natural quanto artificial.

Segundo Baker e Steemers (1998), as ferramentas de projeto simplificadas podem ser subdivididas em: métodos matemáticos; método tabular; e métodos gráficos.

Osmétodosmatemáticossãoprocedimentos de cálculo desenvolvidos através de equações baseadas nas hipóteses físicas, parâmetros geométricos e procedimentos analíticos. Podem ser úteis e relativamente simples para um único ponto, porém lentos e trabalhosos para vários pontos de análise. Como exemplo, podemos citar o cálculo das iluminâncias através do método dos lumens ou do método do fator de luz natural.

O método tabular é baseado na avaliação do fator de luz natural ou a componente do céu através de uma série de parâmetros geométricos.

Os métodos gráficos, bastante utilizados nas fases iniciais de concepção da proposta projetual, são baseados no uso de gráficos, tais como os nomogramas, os transferidores, o diagrama de pontos, os diagramas Waldram, dentre outros (Figura 2). Destacam-se pela facilidade de aplicação e exibição do efeito relativo de diversas variáveis de projeto (MOORE, 1985), porém tem como desvantagem o fato de analisar um único ponto de cada vez, sendo limitado com relação a estudos paramétricos (PEREIRA, 1995).

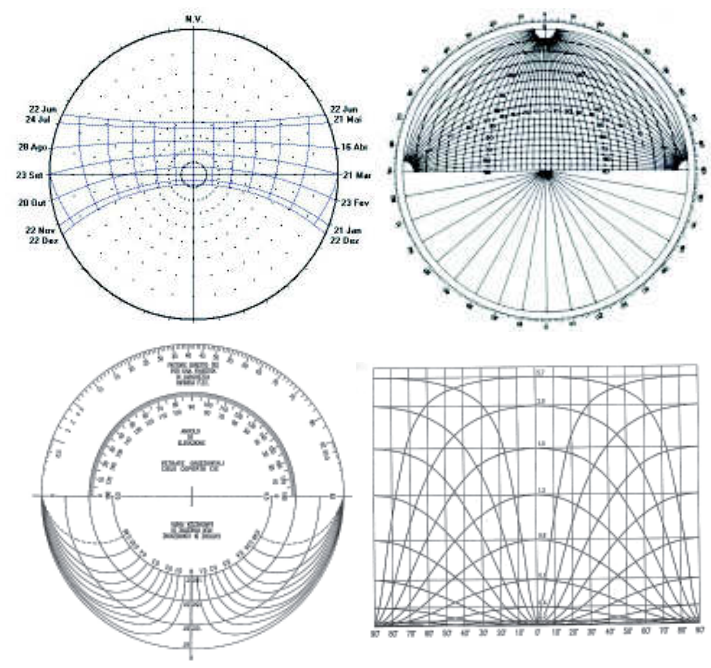

Figura 2 - Exemplos de métodos gráficos (carta solar, transferidor auxiliar, goniômetro BRS e diagrama Waldran, respectivamente)

\section{SIMULAÇÃO COMPUTACIONAL DE ILUMINAÇÃO}

Na década de 50 desenvolveu-se o conceito que a simulação matemática poderia ser aplicada a qualquer processo. Nesse período somente equipamentos de grande porte estavam disponíveis e seu uso estava restrito a poucas instituições de pesquisadores (AMORIM, 1998).

Atualmente, a simulação computacional tem provado - especialmente nas duas ultimas décadas - ser uma ferramenta eficiente para estudar o desempenho ambiental (térmico, luminoso, acústico e energético, dentre outros) dos edifícios. A interação entre os aspectos de projeto, clima, sistemas eletromecânicos e os ocupantes em um edifício é uma tarefa muito complexa e os recursos da simulação possibilitam uma melhor compreensão desses fatores. As ferramentas de simulação permitem apoiar a prática de projeto da arquitetura, possibilitando a realimentação entre a 
tomada de decisões e logo em seguida a avaliação de seu impacto ambiental (CALDAS e NORFORD, 2002).

Segundo Bryan (2002), a simulação computacional da iluminação natural começou na década de 70, com a introdução do LUMEN II (mais tarde LUMEN MICRO) e na metade da década de 80 surgiu o SUPERLITE que podia calcular espaços de formas um pouco mais complexas.

Ubbelohde e Humann

demonstraram que estes programas de simulação computacional encontravam sérios obstáculos - devido à insuficiência de memória e poder computacional baixo - para calcular a luz natural. As tarefas de definir as características de um ambiente, um sistema de aberturas e associar estes dados à distribuição de luminâncias do céu, sobrecarregavam até mesmo os mais avançados computadores da época.

A possibilidade de modelagem das fontes de luz alterou profundamente esse panorama. Inicialmente apenas as fontes de luz diretas eram consideradas, e mesmo assim, apenas uma de cada vez. Pouco a pouco, foram introduzidas fontes mais complexas. Neste contexto as sombras eram "duras", pois os algoritmos preocupavamse exclusivamente com a visibilidade dos objetos e a obstrução decorrente das fontes de luz (BEKAERT, 2003).

Atualmente, existem diversas ferramentas para simulação da iluminação natural que podem ser aplicadas em todas as fases do projeto de arquitetura. A simulação de iluminação tem como principal vantagem a viabilização de estudos quantitativos e qualitativo, permitindo visualizar a aparência do ambiente e a produção de um modelo fotométrico para uma estimativa de suas propriedades luminosas.

Emboracada vez mais complexas, eficientes e sofisticadas, algumas dificuldades são encontradas no uso dessas ferramentas, principalmente no que se refere ao tempo gasto no preparo e edição dos dados relativos à geometria e às propriedades físicas da luz e dos materiais. Entretanto, o processo de simulação, como um todo, tem se tornado mais fácil e interativo permitindo que profissionais e estudantes possam utilizá-los para estudos e projetos, sendo que o tempo despendido é dependente da complexidade do modelo, da implementação do algoritmo de iluminação e da capacidade de processamento do equipamento utilizado.

\subsection{Considerações sobre a aplicação das ferramentas computacionais}

Para avaliar um determinado espaço arquitetônico deve-se, como primeiro passo, elaborar um modelo geométrico tridimensional (objeto-modelo) que represente o espaço a ser estudado (objetoconcreto). Este objeto-modelo deve possuir informações geométricas que definam a representação da realidade em forma de coordenadas cartesianas, $x, y$ e $z$; além de conter informações sobre as propriedades óticas do material de cada superfície e sobre as fontes de iluminação da cena.

O princípio básico é a completa descrição de uma cena 3D, onde se especifica o tamanho e a posição dos objetos e as propriedades dos materiais de todas as superfícies da cena e, fundamentalmente, a posição e as características radiométricas - que se diferencia das fotométricas porque não consideram a percepção humana - das fontes de luz.

A partir destes dados, uma imagem é produzida como se fosse vista por uma câmeravirtual (ou sintética). Ressalte-seque existe uma relação direta entre a qualidade da saída da simulação e a qualidade da elaboração do modelo geométrico.

Assim, o simulador, empregando modelos de iluminação (teorias ou "mecanismos") implementados em algoritmos adequados deve calcular como a luminosidade está distribuída, como cada objeto é iluminado diretamente e indiretamente, segundo o comportamento físico da luz. Exibindo esses resultados das mais variadas formas, inclusive sob a forma de imagens fotorealisticas.

Para a aplicação da simulação computacional os seguintes fatores devem ser considerados: (Figura 3) 


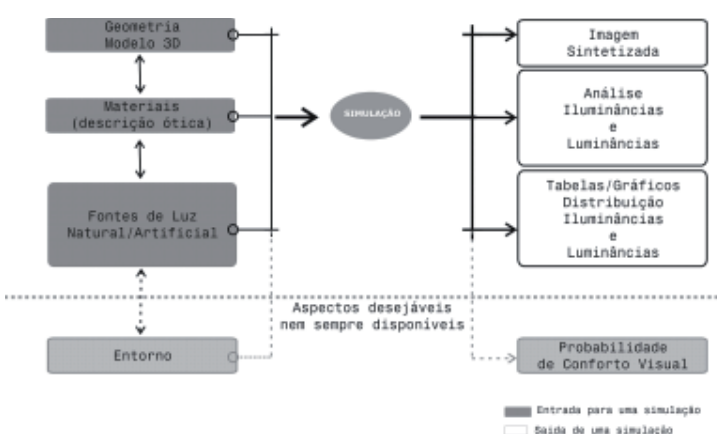

Figura 3 - Fluxograma de fatores envolvidos na simulação computacional da iluminaçãoc

Cabe destacar ainda que há uma diferenciação entre os programas de rendering e as ferramentas de simulação.

Os software de rendering objetivam a obtenção de imagens realísticas do objeto simulado, ignorando a precisão numérica dos resultados. De forma geral, esses programas de rendering têm ênfase na saída gráfica, ou seja, na imagem produzida, visando impactos visuais, não se importando com as técnicas empregadas na sua geração.

Por outro lado, os programas para simulação são propostos para reproduzir o comportamento da luz e sua interação com os materiais através de cálculos complexos, fundamentados no fenômeno físico da luz na cena. A sua principal tarefa não é mostrar uma visão realística do projeto arquitetônico ao leigo, mas permitir a avaliação da qualidade e adequação da solução proposta através de imagens sintéticas e informações numéricas (INANICI, 2001).

Para isso, essas ferramentas utilizam algoritmos chamados de "iluminação global", que segundo Ehrlich (2002), simulam as propriedades físicas e comportamentais da luz, em escalas macro (propagação da luz da fonte ao observador) e em escala micro (interações da luz com as características das superfícies).

As principais aproximações algorítmicas de predição da luz natural baseiam-se nos modelos de iluminação global que existem basicamenteem duas vertentes. Os métodos baseados na cena e que são independentes do ponto de vista do observador - ou simplesmente "scene-based" como o RADIOSITY ${ }^{\mathrm{d}}$. E os métodos baseados na imagem e que são dependentes do ponto de vista do observador - "image-based" como o RAY TRACINGe. Ambos são capazes de produzir resultados numéricos além de imagens de alta qualidade, ou seja, avaliações quantitativas e qualitativas da luz natural no espaço arquitetônico.

A simulação da luz natural deve utilizar algoritmos devidamente validados. A validação é um processo essencial, pois visa averiguar se os resultados obtidos através da simulação são compatíveis, a partir de uma relação entre os quantitativos físicos disponíveis e os parâmetros de simulação da luz natural.

Segundo Baker et al. (1993), basicamente existem dois níveis de validação na simulação da luz natural:

Avaliar se as iluminâncias são as mesmas que no edifício real, onde tudo deve ser similar ao que foi simulado. Devido a limitações das ferramentas, esse edifício deve ser uma célula de teste sob um céu artificial; e

Previsão da performance da iluminação natural de um edifício real para condições climáticas reais. Isto requer ajustes dos resultados da simulação e uma definição estatística do desempenho.

\subsection{Projetação arquitetônica e simulação da iluminação}

A simulação deve estar inserida paralelamente às etapas do processo de projeto nas quais se definem o "partido" ou o "risco" inicial. Alguns ajustes podem ser adotados com novas entradas de dados e então processados novamente, até que a solução satisfaça os objetivos pretendidos. Então, volta-se ao processo de elaboração do projeto já em fase do anteprojeto e, portanto, elaborado com um grau de certeza suficiente para seguir para a fase seguinte (figura 4 ). 


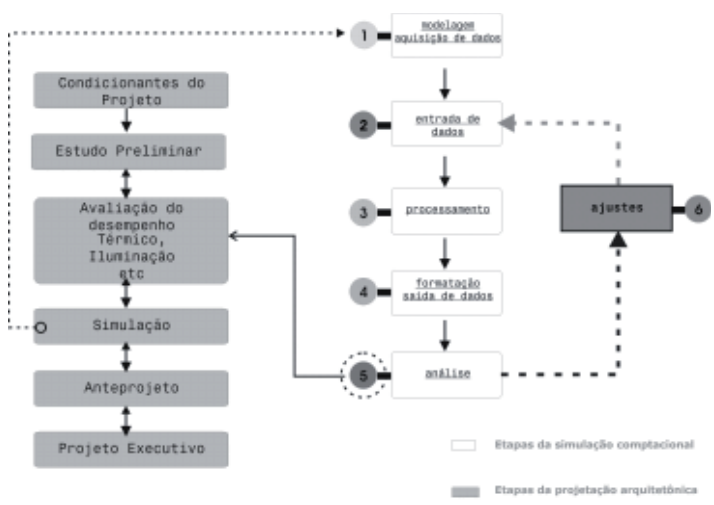

Figura 4 - Fluxograma de Projeto Arquitetônico apoiado por simulação computacional $^{\mathrm{f}}$

$\mathrm{O}$ projeto de arquitetura pode-se beneficiar com a técnica de simulação da luz natural, com a qual é possível gerar imagens preditivas de espaços que ainda não foram construídos. Podem-se avaliar previamente as condições de conforto visual, computando os diversos níveis de iluminação em diferentes pontos do ambiente, possibilitando que se façam os ajustes necessários. Além disso, pode-se simular o projeto de iluminação artificial conjugado ao da luz natural, possibilitando melhora significativa na eficiência energética do edifício.

Apesar dessas facilidades, não há uma avaliação precisa de quantos profissionais consideram os métodos de cálculo da luz natural como parte das tarefas a serem elaboradas no projeto arquitetônico. Até 1992, na Europa, somente $15 \%$ dos arquitetos se ocupavam dos assuntos da luz natural em seus projetos (IEA, 2000).

Dez anos depois, outra pesquisa mostrou que $91 \%$ responderamqueincluemaspectos da luz natural nos projetos de edifícios, sendo que, entre estes, $79 \%$ utilizam a simulação computacional, demonstrando forte tendência de crescimento da sua aplicação como apoio qualitativo ao processo de projeto (MAHDAVI, 2003).

Quanto à realidade brasileira, em estudos sobre a simulação computacional, verificou-se uma baixa aceitação do uso das ferramentas de simulação. Isto se deve, em primeiro lugar, à falta de legislação específica para a aplicação dos conceitos de eficiência energética em novos edifícios.
Em segundo lugar, existe uma falta de confiança na aplicabilidade destes programas de simulação na realidade brasileira, em termos de clima e tipologia das edificações locais (MENDES et al., 2001).

Vale ressaltar que o conceito de integração da simulação na projetação do edifício, não é suficiente per si. Esta barreira pode ser superada através da elaboração de ferramentas adequadas ao fluxo de trabalho do arquiteto, assim como a inclusão de temas de simulação computacional na formação dos futuros arquitetos.

\subsection{Estudos comparativos de ferramentas para simulação computacional}

Muitos estudos têm sido desenvolvidos a cerca do uso das ferramentas computacionais para a simulação da iluminação natural, tanto em âmbito internacional, onde a maioria dos programas voltados para esse enfoque é produzido, quanto em âmbito nacional, existindo vários estudos que têm descrito a aplicação desses programas e discutido a validação dos mesmos, que é uma condição preliminar para a garantia da precisão dos resultados.

Tais pesquisas fazem uma análise da usabilidade, características e, principalmente, os recursos disponíveis para a simulação quantitativa e qualitativa da luz, que podem auxiliar o projetista na aplicação desses recursos no ato de projetar em arquitetura.

Nesse sentido, destacam-se as pesquisas realizadas no IEA - International Energy Agency - da LBL - Lawrence Berkeley National Laboratory - que desenvolve trabalhos na área de conforto ambiental, dentre os quais a iluminação, publicando estudos sobre algoritmos, métodos e ferramentas, além de terem desenvolvido alguns dos programas de simulação utilizados pela comunidade científica, tais como o ADELINE e o RADIANCE.

Lima (2003) destaca que muitos trabalhos publicados visam também comparar as diversas ferramentas de simulação de 
iluminação quando utilizadas no processo de projeto de arquitetura, tais como, Roy (2000) e Ashmore e Richens (2001) que analisaram os programas ADELINE 2.0 NT, LIGHTSCAPE 3.1.1, MICROSTATION 7.1 e RADIORAY 2.0 quanto a precisão e facilidade de uso; e Khodulev \& Kopylov (2002) e Ubbelohde e Humann (1998), que fazem um estudo comparativo entre programas analisando as interfaces, a exatidão da descrição física das fontes luminosas e dos materiais, o modelo de iluminação global utilizado, a acurácia da simulação e a capacidade do usuário de monitorar e controlar a precisão da simulação.

No Brasil, alguns trabalhos têm procurado avaliar e comparar diversos programas de iluminação natural, inclusive do ponto de vista do uso dos arquitetos e projetistas em geral. Christakou (2004) em seu trabalho, compara 4 ferramentas para a simulação da iluminação natural - DESKTOP RADIANCE, LIGHTSCAPE, RAYFRONT e RELUX VISION - escolhidos devido à sua maior flexibilidade, precisão (validação) e atualidade dos mesmos; e Lima (2003) faz uma análise dos programas LIGHTSCAPE, LUMEN MICRO E DESKTOP RADIANCE, avaliando sua aplicação no desenvolvimento de projetos de iluminação natural e artificial (Figura 5).

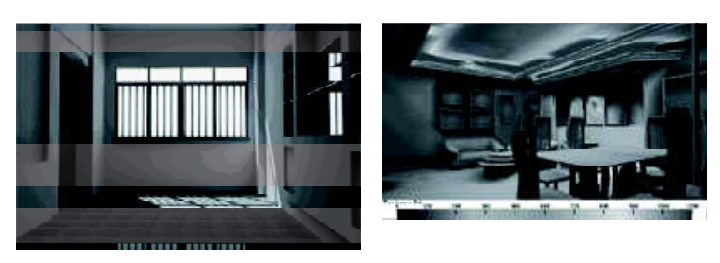

Figura 5 - Simulação de iluminação: imagem texturizada e em escala de cinza, respectivamente ${ }^{\mathrm{g}}$

\section{CONCLUSÃO}

As decisões tomadas pelo arquiteto nos estágios iniciais do projeto têm impacto decisivo no desempenho luminoso da edificação. Considerando que a projetação arquitetônica é um processo seqüencial de tomadas de decisões, a definição das propriedades e comportamentos impostos nas fases iniciais tendem a perdurar e a influenciar nas decisões posteriores e assim por diante.

Dentre os diversos instrumentos existentes para o cálculo e avaliação da iluminação natural, destaca-se a possibilidade do uso de modelos físicos em escala reduzida, que por serem de maior utilização por parte dos projetistas, pode auxiliar nos estudos de iluminação e na determinação das melhores soluções de projeto. No entanto, como discutido anteriormente, requerem tempo para execução e detalhamento, principalmente com relação a ambientes complexos.

Das ferramentas consideradas simplificadas, as mais utilizadas são as de aplicação gráfica, tais como a carta solar, a máscara de sombra e os transferidores, que permitem determinar com facilidade a incidência da radiação solar nos ambientes e os elementos de controle da insolação; e os métodos matemáticos, que permitem calcular a quantidade de iluminação. Embora bastante utilizados, são limitados em relação aos estudos qualitativos da iluminação, pois não permitem uma percepção visual do espaço avaliado.

A simulação computacional, no entanto, permite esse tipo de análise, favorecendo a realização de estudos mais aprofundados do uso da luz natural, mas depende basicamente da disponibilidade de tempo, do poder de processamento e da confiabilidade das ferramentas que $\mathrm{o}$ arquiteto dispõe para realizar a tarefa da integração da luz natural ao seu projeto arquitetônico. A interatividade na experimentação de alternativas de projeto é um dos principais objetivos a ser alcançado para lidar com este complexo conjunto de aspectos da luz natural.

Em geral, é possível concluir que os programas devem ser usados com muita cautela, pois os cálculos de iluminação são muito sensíveis à qualidade dos dados de entrada: descrição das fontes de luz, fotometria dos materiais, geometria da edificação (objeto-modelo) e dos parâmetros de simulação.

Outro ponto a ser destacado é a 
necessidade cada vez maior da ampliação desses conhecimentos na prática projetual. A simulação da iluminação ganha força e sinergia quando existe um processo de concepção arquitetônica em ambiente computacional fundamentado na modelagem geométrica tridimensional, visto que uma das etapas mais trabalhosas da simulação é exatamente a construção do modelo geométrico. Assim, a simulação pode influenciar nas decisões arquitetônicas e sucessivamente voltar a alimentar e influenciar na simulação. Isso pode ser conseguido através da ampliação do uso dessas ferramentas no ensino das disciplinas de conforto integradas às de projeto, de forma a disseminar as informações no ambiente acadêmico, e também na prática profissional. Para isso, diversos grupos de pesquisa nas universidades $^{\mathrm{h}}$ têm trabalhado com o intuito de avaliar a qualidade ambiental do projeto de arquitetura, estudando as novas tecnologias disponíveis e sua aplicação no desenvolvimento de projetos.

\section{REFERÊNCIAS BIBLIOGRÁFICAS}

ABNT - ASSOCIAÇÃO BRASILEIRA DE NORMAS TÉCNICAS, Rio de Janeiro. NBR 15215-3: iluminação natural - Partes 2, 3 e 4. Rio de Janeiro: ABNT, 2005.

AMORIM, Claudia Naves David. Desempenho térmico de edificações e simulação computacional no contexto da arquitetura bioclimática: Estudo de casos na região de Brasília. 1998. Dissertação (Mestrado em Arquitetura e Urbanismo), Programa de Pós-Graduação em Arquitetura e Urbanismo, Universidade de Brasília, 1998.

ASHMORE, J., RICHENS, P. Computer simulation in daylight design: a comparison. March, 2001.

BAKER, N. \& STEEMERS, K. Daylight design of buildings. London: James and James Editors, 1998.

BAKER, N.; FANCHIOTTI, A.;STEEMERS, Koen. Daylighting in architecture: A European Reference Book. Londres: James and James Editors, 1993.
BEKAERT, Philippe et al. Advanced global illumination. Bélgica: Ed. AK Peters, 2003.

BRYAN, Harvey; AUTIF, Sayed M. Lighting/daylighting analysis: a comparasion. Proceedings of the 27th National Passive Solar Conference, American Solar Energy Society, USA: R. Campbell-Howe editor, 2002.

CALDAS, Luiza G., NORFORD, Leslie K. A design optimization tool based on a genetic algorithm. Em "Automation in Construction", 2002 págs 173-184.

CHRISTAKOU, E., D. Simulação da luz natural aplicada ao projeto de arquitetura. 2004. Dissertação (Mestrado em Arquitetura e Urbanismo) - Programa de Pós-Graduação em Arquitetura e Urbanismo, Universidade de Brasília, 2004.

ERLICH, Charles K. Computer aided perception: a method to evaluate the representation of glare in computer graphics imagery. ,2002. Dissertação (Mestrado) University of California, 2002.

IEA - International Energy Agency. Daylight in buildings: a source book on daylighting systems and components. A report of IEA Task 21/ Ecbcs, Annex 29. 2000.

INANICI, Mehlika N. Application of the state-of-the-art computer simulation and visualization in architectural lighting research. In: Proceedings of the Building Simulation- Seventh International IBPSA Conference. Rio de Janeiro: IBPSA, 2001.

KHODULEV, A. B., KOPYLOV, E. A. Physically accurate lighting simulation in computer graphics software. Disponível na Internet: http://rmp.kiam.ru/articles/ pals/introcuction.htm. Acesso em: 29/10/2002.

LIMA, Thais Borges Sanches. Uso da simulação computacional em projetos de iluminação interna. 2003. Dissertação (Mestrado em Arquitetura e Urbanismo). Programa de Pós-Graduação em Arquitetura e Urbanismo, Universidade Federal da Bahia, 2003. 
MAHDAVI, A et al. An inquiry into the building performance simulation usage by architects in Austria. In: Proceedings of the Eighth International IBPSA Conference. Eindhoven: IBPSA, 2003.

MENDES, Nathan, LAMBERTS, Roberto, CUNHA NETO, José A. Bellini. Building simulation in Brasil. In: Proceedings of the Seventh International IBPSA Conference. Rio de Janeiro: IBPSA, 2001.

MOORE, F. Concept and practice of architectural daylighting. New York: Van Nostrand Reinhold Company, 1985.

PEREIRA, Fernando O. R. Iluminação natural no ambiente construído. Apostila do curso, ministrado no III ENCAC. Gramado: Antac, 1995.

ROY, Geoffrey G. A comparative study of lighting simulation packages suitable for use in architectural design. Murdoch University - School of Engineering, 2000.

UBBELOHDE, Susan M.; HUMANN, Christian. Comparative evaluation of four daylighting software programs. Berkeley: American Council for an Energy-Efficient Economy; University of California, 1998.

\section{NOTAS}

a Embora estas ferramentas possam também ser aplicadas para o projeto de iluminação artificial, aqui será tratada apenas a iluminação natural, foco deste artigo.

b Foto: Paulo Marcos Paiva de Oliveira

c Fonte: Cristakou, 2004

d O método de Radiosity se baseia na discretização das superfícies em retalhos (células) de tamanho uniforme e a energia trocada entre estes retalhos é computada de uma maneira totalmente independente do ponto de vista do observador.

e Refere-se ao Ray Tracing na sua concepção atual que adota uma estratégia Monte Carlo no traçado dos raios por amostragens e que se baseia em calcular a interação no trajeto dos raios de luz com a geometria.

f Christakou, 2004

g Lima, 2003

$\mathrm{h} \quad$ Dentre os grupos de pesquisa existentes, o grupo "Qualidade Ambiental e Iluminação Natural no Espaço Construído", da Universidade de Brasília, tem uma linha de pesquisa em "Simulação Computacional no Espaço Construído". http:// www.unb.br/fau/qualilumi/ 